\title{
CAMBIOS ESTACIONALES DEL MANTILLO EN EL SUELO DE UN BOSQUE TROPICAL CADUCIFOLIO Y UNO SUBCADUCIFOLIO EN CHAMELA, JALISCO, MEXICO
}

\author{
Angelina Martinez Yrizar y Jose Sarukhan \\ Centro de Ecología \\ Universidad Nacional Autónoma de México \\ Apartado Postal 1354 \\ C.P. 83000 \\ Hermosillo, Sonora, México
}

\begin{abstract}
RESUMEN
Se analizaron los cambios temporales del mantillo en un bosque tropical caducifolio y uno subcaducifolio en la Estación de Biología, Chamela, Jalisco, México. Colectas mensuales de mantillo mostraron que el grosor promedio de la capa de mantillo es mayor en el sitio de bosque subcaducifolio y que su tiempo de recambio es más rápido. La variación temporal del mantillo exhibió un patrón estacional. En ambos sitios, el mantillo tendió a acumularse por la caída de hojarasca durante el perlodo de sequia, y a disminuir por descomposición durante el período de lluvias.
\end{abstract}

\begin{abstract}
The present study reports on the seasonal variation of standing crop litter in a tropical deciduous and a semideciduous forest in Chamela, Jalisco, México. The amount of surface litter was, on average, higher in the semideciduous forest, where the turnover rate of standing crop litter was faster. The temporal variation in the mean amount of standing crop litter exhibited a marked seasonal pattern. Surface litter tended to accumulate during the dry season when litterfall was at its maximum, and decreased considerably as a result of decomposition during the rainy season.
\end{abstract}

\section{INTRODUCCION}

El mantillo (en inglés "surface litter" ó "standing crop litter") es la capa de residuos orgánicos que yace sobre la superficie del suelo de las comunidades vegetales. La profundidad y complejidad del mantillo en un sitio dado, están determinadas por un balance entre la caída de hojarasca y su destrucción por agentes físicos, reacciones químicas y actividad de los organismos del suelo (Facelli y Pickett, 1991). El mantillo constituye uno de los principales sitios de almacenamiento de carbono y elementos minerales en el suelo de algunos ecosistemas (Vogt et al., 1986). Es también un componente dinámico fundamental en los procesos de flujo de energía y circulación de nutrientes dentro del 
ecosistema (Jordan, 1985; Vogt et al., 1986). Algunos autores han reconocido recientemente, que el mantillo puede afectar la organización y estructura de las comunidades vegetales por su efecto en la germinación de semillas, la sobrevivencia de plántulas y el desarrollo de retoños vegetativos (Sydes y Grime, 1981a,b; Vázquez Yanes et al., 1990; Facelli y Pickett, 1991).

El presente estudio forma parte de una investigación a largo plazo sobre la estructura y funcionamiento de un ecosistema natural de bosque tropical caducifolio y subcaducifolio en Chamela, Jalisco, México. Complementa los resultados de estudios previos que describen los patrones estacionales de caida y descomposición de hojarasca en los sitios de estudio (Martínez Yrízar, 1980; Vizcaíno, 1983; Martínez Yrízar y Sarukhán, 1990). Aquí analizamos los cambios temporales de la capa de mantillo en el suelo con el objeto de evaluar su descomposición y significado en la dinámica de la hojarasca dentro del ecosistema.

\section{SITIOS DE ESTUDIO Y METODOS}

El estudio se realizó en la Estación de Biología Chamela, del Instituto de Biología, Universidad Nacional Autónoma de México, a $19^{\circ} 30^{\prime} \mathrm{N}, 105^{\circ} 03^{\prime} \mathrm{W}$, cercana al poblado de Chamela, en la costa de Jalisco, México (para una descripción más detallada del sitio ver Bullock, 1988). Es una reserva de 1600 ha, en relieve de lomeríos con pendientes de $21^{\circ}$ a $34^{\circ}$ (Bullock, 1988). Los suelos son entisoles jóvenes poco desarrollados en substratos de riolitas y basaltos ( $E$. Solis, com. pers.).

El clima es cálido y estacionalmente seco. $84 \%$ de la precipitación promedio anual (707 mm, 1977-1988) cae entre julio y octubre. La temperatura media anual es de $24.9^{\circ} \mathrm{C}$. (Bullock, 1988). La variación en la precipitación anual es alta debido a la ocurrencia de tormentas de orígen ciclónico que varían en duración e intensidad de un año a otro (GarcíaOliva, 1992).

El tipo de vegetación predominante es el bosque tropical caducifolio de Rzedowski (1978), excepto por pequeñas áreas de bosque tropical subcaducifolio a lo largo de los arroyos y en suelos más profundos. Se han reconocido casi 780 especies de plantas herbáceas y arbóreas en el área de la Estación (Lott, 1985). Las leguminosas y euforbiaceas son las familias más diversas. La florística y estructura de la vegetación se discuten en Lott et al. (1987).

Se eligieron dos sitios de estudio.

1) Sitio Arroyo, con una extension de 0.42 ha, de pendiente ligera (menos de $5^{\circ}$ )y a $60 \mathrm{~m}$ de altitud. El suelo es migajón arenoso, café obscuro y de $80 \mathrm{~cm}$ de profundidad. La vegetación es un bosque tropical subcaducifolio (Rzedowski, 1978) de $15 \mathrm{~m}$ de altura. Presenta 64 especies de árboles con un diámetro a la altura del pecho (DAP) mayor de $3.0 \mathrm{~cm}$. Cordia elaeagnoides DC. y Caesalpinia eriostachys Benth. son las especies más abundantes, constituyendo $32 \%$ de los $22.5 \mathrm{~m}^{2}$ ha-1 de área basal total (A. Pérez Jiménez, com. pers.). En este sitio aproximadamente $75 \%$ del dosel de vegetación permanece sin follaje durante la estación seca.

2) Sitio Ladera, con una extensión de 1.0 ha y de pendiente pronunciada $\left(20^{\circ}-40^{\circ}\right)$ se localiza a una altitud de $90 \mathrm{~m}$. El suelo es somero (menos de $40 \mathrm{~cm}$ de profundidad), con textura de arena migajosa y color café-amarillo obscuro. La vegetación es un bosque 
tropical caducifolio de $6 \mathrm{~m}$ de alto. Presenta 53 especies arboreas (DAP $>3.0 \mathrm{~cm}$ ). C. eriostachys y Apoplanesia paniculata Presl son las principales especies que constituyen $20 \%$ de los $14 \mathrm{~m}^{2} \mathrm{ha}^{-1}$ de área basal total (A. Pérez Jiménez, com. pers.). Con excepción de algunos individuos de especies helibfilas, como Jacquinia pungens A. Gray y Coccoloba liebmannii Lindau, la gran mayoría de los individuos tiran todo el follaje en la época de sequía.

Para determinar la cantidad promedio de mantillo en pie, se muestreó aleatoriamente el suelo de cada sitio usando un anillo metálico de $20 \mathrm{~cm}$ de diámetro $\left(0.0314 \mathrm{~m}^{2}\right)$. Toda la hojarasca contenida en el área del anillo, se recogió manualmente y se guardó en bolsas de papel. Posteriormente, las muestras se secaron en un horno ventilador a $80^{\circ} \mathrm{C}$ hasta alcanzar peso constante. Se eligieron 29 puntos de colecta en el sitio Arroyo y 65 en el sitio Ladera. El número total de muestras colectadas por sitio se determinó tomando en cuenta la extensión de los mismos. Las colectas se efectuaron mensualmente durante un año a partir de marzo de 1978.

\section{RESULTADOS Y DISCUSION}

La cantidad promedio de mantillo en Chamela, para el año de estudio, fue significativamente mayor en el sitio Arroyo (10.4 \pm 0.27 (error estandar) $\mathrm{Mg} \mathrm{ha}^{-1}$ vs 9.0 $\pm 0.29 \mathrm{Mg} \mathrm{ha}^{-1}$, en el sitio Ladera; $\left.\mathrm{F}=12.92, \mathrm{P}=0.0001\right)$. Estos valores son mayores que los señalados por Patiño (1990) para otros sitios similares dentro de la Estación (intervalo de 7.5 a $8.2 \mathrm{Mg} \mathrm{ha}^{-1}, \mathrm{n}=7$ ). Esta diferencia posiblemente se explica si se considera que en nuestro trabajo, solamente se tamizaron las muestras, para corregir la adición de suelo mineral, que contamina el mantillo. Patiño (1990) no sólo tamizó sus muestras, sino que además las incineró en una mufla, restando también su contenido mineral.

El análisis de la variación temporal de mantillo en ambos sitios, mostró un patrón estacional estadísticamente significativo; $F=71.34, P=0.01$, (Fig. 1). Al inicio del estudio, el mantillo aumentó hasta alcanzar un valor máximo en mayo-julio. Posteriormente, la cantidad promedio disminuyo hasta un valor mínimo que coincidió con el término de la estación de lluvias en octubre-noviembre. El porcentaje de pérdida de mantillo durante este tiempo fue de $54 \%$ y $67 \%$ para los sitios Ladera y Arroyo, respectivamente (Fig. 1).

Para otros sitios de bosque caducifolio y subcaducifolio en Chamela, Patiño (1990) también registró una marcada estacionalidad en el grosor del mantillo. Cambios estacionales en otras comunidades tropicales climáticamente similares a las de Chamela han sido registrados por Madge (1965) y Hopkins (1966) en Nigeria, Lugo et al: (1978) y Cintrón y Lugo (1990) en Puerto Rico y Swaine et al. (1990) en Ghana.

No se encontró una correlación estadísticamente significativa entre el grosor promedio mensual del mantillo y la cantidad mensual de lluvia en Chamela. Esto se debe a que el grosor del mantillo en un tiempo dado, no depende únicamente de la tasa a la cual se pierde por descomposición, sino también de la cantidad de hojarasca que se añade al suelo en ese mismo intervalo (Vogt et al., 1986; Facelli y Picket, 1991). En Chamela, por ejemplo, el mantillo disminuye muy rápidamente durante los meses de lluvia, debido principalmente a la descomposición de las hojas (Martínez Yrízar, 1980). Simultáneamente, el viento y las lluvias más intensas, ocasionan un aumento en el matillo por la caída de una gran cantidad de hojas y fragmentos de ramas muertas en pie. 


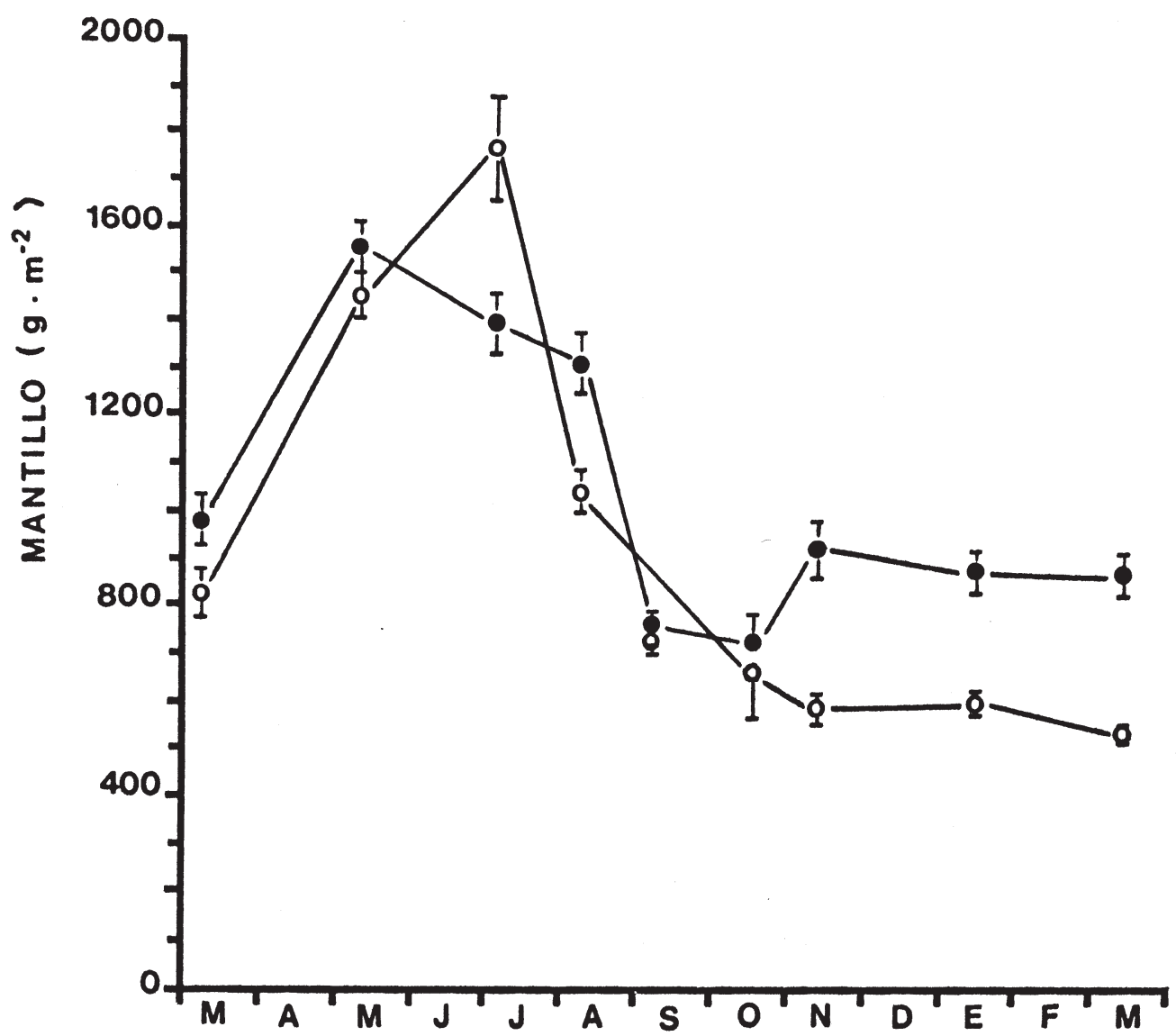

Fig. 1. Variación temporal del mantillo ( $\mathrm{g} \mathrm{m}^{-2}$, peso seco, \pm 1 error estandar) en el bosque tropical caducifolio (Sitio Ladera, o - o) y en el bosque tropical subcaducifolio (Sitio Arroyo, • - •) en la Estación de Biología, Chamela, Jalisco, México. El eje de las abscisas indica el tiempo en meses, iniciando en marzo de 1978.

La descomposición del mantillo se puede resumir calculando su tiempo de recambio en el suelo $(T)$ usando la ecuación $T=M / H$, donde $M$ es la cantidad promedio anual de mantillo en pie y $\mathrm{H}$ es la caída anual de hojarasca. Este valor, que es un promedio anual, debe tomar en cuenta las siguientes condiciones: 1) que las tasas de descomposición varían de la estación seca a la húmeda (Martínez Yrízar, 1980, Swaine et al., 1990) y 2) que el mantillo ha alcanzado un estado estable en el ecosistema (Olson, 1963). Los valores de $\mathrm{T}$ han sido muy útiles para establecer comparaciones entre sitios, y entre las distintas fracciones del mantillo, i.e. ramas, hojas, flores, etc. (Swaine et al., 1990). 
En nuestro estudio, $T$ se calculó utilizando los datos de producción anual de hojarasca $(H)$ de Martínez Yrízar y Sarukhán (1990): $\mathrm{H}_{\text {Aroyo }}=6.4 \mathrm{Mg} \mathrm{ha}^{-1}$ año-1 $^{-1} \mathrm{H}_{\text {Ladera }}=$ 4.3 $\mathrm{Mg} \mathrm{ha}^{-1}$ año-1. Para el sitio Arroyo T fue de 1.6 años, menor que el valor del sitio Ladera, 2.1 años. Esto significa que a pesar de que el mantillo en el sitio Arroyo es más grueso, y que la adición de hojarasca es significativamente mayor, su tiempo de recambio es más corto que en el sitio Ladera.

En otras localidades de bosque caducifolio en el trópico, los valores de $T$ varían desde sitios donde el recambio ocurre en menos de un año, por ejemplo en Ibadan, Nigeria (Madge, 1965), hasta el extremo donde el recambio ocurre en tres años o más, como en el bosque de Guánica, Puerto Rico (Lugo y Murphy 1986, Cintrón y Lugo, 1990).

Diferencias entre sitios en el tiempo de residencia del mantillo en el suelo naturalmente implican diferencias en las características de circulación de nutrientes de un sitio a otro. Si T es un indicador de la tasa a la cual el reservorio de nutrientes en el suelo se abastece por descomposición, éste puede ser crítico en determinar si ocurrirá una mayor inmovilización de nutrientes por actividad microbiana (concentrando así los nutrientes en el suelo) o si ocurrirá una mayor absorción de nutrientes por las raíces de las plantas. El balance entre estos dos procesos estará afectado por la eficiencia de captación de los nutrientes por las raíces y la masa microbiana, así como por las propiedades del suelo (Vogt et al., 1986).

La interpretación de los mecanismos que afectan la dinámica de nutrientes en el suelo, se basa en estudios sobre los cambios espaciales y temporales del mantillo. Estos cambios deben integrarse a datos sobre la actividad de los organismos descomponedores, el crecimiento radicular y un inventario total de los nutrientes, para asi poder inferir acerca de las características del ciclaje de nutrientes en el ecosistema.

\section{AGRADECIMIENTOS}

Agradecemos especialmente a los Maestros en Ciencias Ana Mendoza, Mónica Vizcaíno y Alfredo Pérez Jiménez, a los Biólogos Arturo Solís Magallanes, José Luis Alvarado y Silvia Núnez su apoyo y colaboración en varias etapas del estudio. Agradacemos al Dr. Alberto Búrquez, Dr. Luis Eguiarte y dos revisores anónimos sus valiosas correcciones al manuscrito original. Este trabajo se realizo con financiamiento del Consejo Nacional de Ciencia y Tecnología, México. El Instituto de Biología, Universidad Nacional Autónoma de México, brindó además todas las facilidades para trabajar en sus instalaciones de la Estación de Biología Chamela.

\section{LITERATURA CITADA}

Bullock, S. H. 1988. Rasgos del ambiente físico y biológico de Chamela, Jalisco, Mexico. Folia Entomológica Mexicana 77: 5-17.

Cintrón, B. B. y A. E. Lugo. 1990. Litterfall in a subtropical dry forest: Guanica, Puerto Rico. Acta Científica 4: $37-49$.

Facelli, J. M. y S. T. A. Pickett. 1991. Plant litter: its dynamics and effects on plant community structure. Botanical Review 57: 1-32. 
García Oliva, F. F. 1992. Erosión y pérdida de nutrientes del suelo en una cronosecuencia de uso agropecuario en un ecosistema tropical estacional mexicano. Tesis Doctoral. Centro de Ecología, Universidad Nacional Autónoma de México. México, D.F. 154 pp.

Hopkins, B. 1966. Vegetation of the Olokemeji forest, Nigeria. IV. The litter and soil with special reference to their seasonal changes. Journal of Ecology 54: 687-703.

Jordan, C. F. 1985. Nutrient cycling in tropical forest ecosystems. John Wiley. Chichester. 189 pp.

Lott, E. J. 1985. Listados florísticos de México. III. La Estación de Biología, Chamela, Jalisco. Instituto de Biología. Universidad Nacional Autónoma de México. México, D.F. 47 pp.

Lott, E. J., S. H. Bullock y J. A. Solis-Magallanes. 1987. Floristic diversity and structure of upland and arroyo forests of coastal Jalisco. Biotropica 19: 228-235.

Lugo, A. E. y G. Murphy. 1986. Nutrient dynamics of a Puerto Rican subtropical dry forest. Journal of Tropical Ecology 2: 55-72.

Lugo, A. E., J. A. González-Liboy, B. Cintrón y K. Dugger. 1978. Structure, productivity and transpiration of a subtropical dry forest in Puerto Rico. Biotropica 10: 278-291.

Madge, D. S. 1965. Leaf fall and litter disappearance in a tropical forest. Pedobiologia 5: 273-288.

Martínez Yrízar, A. 1980. Tasas de descomposición de materia orgánica foliar de especies arbóreas de selvas en clima estacional. Tesis de Licenciatura. Facultad de Ciencias. Universidad Nacional Autónoma de México. México, D.F. 126 pp.

Martínez Yrizar, A. y J. Sarukhán.1990. Litterfall patterns in a tropical deciduous forest in México over a five-year period. Journal of Tropical Ecology 6: 433-444.

Olson, J. O. 1963. Energy storage and the balance of producers and decomposers in ecological systems. Ecology 44: 322-331.

Patiño, M. C. 1990. Variación espacial y temporal de la capa de hojarasca (mantillo) en una selva baja caducifolia en Chamela, Jal. México. Tesis de Licenciatura. Facultad de Ciencias. Universidad Nacional Autónoma de México. México, D.F. 78 pp.

Rzedowski, J. 1978. Vegetación de México. Limusa. México, D.F. 431 pp.

Swaine, M. D., D. Lieberman y J. B. Hall. 1990. Structure and dynamics of a tropical dry forest in Ghana. Vegetatio 88: 31-51.

Sydes, C. y J. P. Grime. 1981a. Effects of tree leaf litter on herbaceous vegetation in deciduous woodland. I. Field investigations. Journal of Ecology 69: 237-248.

Sydes, C. y J. P. Grime. 1981b. Effects of tree leaf litter on herbaceous vegetation in deciduous woodland. II. An experimental investigation. Journal of Ecology 69: 249-262.

Vázquez Yanes, C., A. Orozco Segovia, E. Rincón, M. E. Sánchez Coronado, P. Huante, J. R. Toledo y V. L. Barradas. 1990. Light beneath the litter in a tropical forest: effect on seed germination. Ecology 71: 1952-1958.

Vizcaíno, M. 1983. Patrones temporales y espaciales de producción de hojarasca en una selva baja caducifolia en la costa de Jalisco, México. Tesis de Maestría. Facultad de Ciencias. Universidad Nacional Autónoma de México. México, D.F. 137 pp.

Vogt, K. A., C. C. Grier y D. J. Vogt. 1986. Production, turnover and nutrient dynamics of above-ground detritus of world forests. Advances in Ecological Research 15: 303-377. 